\section{Bei Asthmatikern ist vielleicht schon das erste Gläschen zu viel}

\author{
Jeder dritte Asthmatiker berichtet, dass Wein oder andere alkoholi- \\ sche Getränke gelegentlich eine Exazerbation verursachen. Sulfite \\ und Salicylate werden als mögliche Auslöser diskutiert. Histamin \\ scheidet dagegen als Ursache von Unverträglichkeiten auf Wein aus.
}

tionen im Zusammenhang mit anderen sulfithaltigen Nahrungsmitteln. 36 Patienten machten entsprechende Angaben, was eine hochsignifikante Assoziation (6,5-faches Risiko), aber eben noch keinen kausalen Beweis bedeutet. Ein anderer Kandidat sind die im Wein enthaltenen Salicylate. Hier ist der Zusammenhang weniger deutlich: Patienten mit durch Acetylsalicylsäure induziertem Asthma hatten ein 2,9-fach erhöhtes Risiko. Aber auch dies beweist noch nichts.

Dass Vorsicht angebracht ist, zeigt eine andere Studie, die den Zusammenhang zwischen dem Histamingehalt des Weins und Unverträglichkeitsreaktionen untersuchte. Gisele Kanny und Mitarbeiter vom Zentralkrankenhaus in Nancy, Frankreich, führten bei 16 Patienten mit Weinunverträglichkeit (alle mit chronischer Urtikaria und typischen „Histamin-Reaktionen" auf Weinkonsum) eine doppelblinde Provokation mit zwei verschiedenen Weinsorten durch.

Obwohl der Histamingehalt beider Weine deutlich differierte $(0,4 \mathrm{mg} / \mathrm{l}$ oder $13,8 \mathrm{mg} / \mathrm{l})$, gab es keine Unterschiede hinsichtlich der beobachteten Symptomatik. Auch die Serumspiegel von Histamin und seiner Abbauprodukte waren nach $\mathrm{Ge}$ nuss der beiden Weine kaum verschieden. Paradoxerweise hatten die Teilnehmer unmittelbar nach dem Genuss des histaminarmen Weins die höchsten Serumspiegel.

\section{Fazit}

Da Wein eine Vielzahl von Inhaltsstoffen enthält, ist es schwer, die Ursache von Unverträglichkeitsreaktionen endgültig „dingfest“ zu machen.

rme

Vally H et al. Alcoholic drinks: Important triggers for asthma? J Allergy Clin Immunol 2000; 105: 462-7

Kanny G et al. No correlation between wine intolerance and histamine content of wine. J Allergy Clin Immunol 2001; 107: 375-8 